\title{
Dithiol-Induced Oligomerization of Thiol-Protected Gold Nanoclusters
}

\author{
Karolina Sokolowska, Eero Hulkko, ${ }^{\circledR}$ Lauri Lehtovaara,* and Tanja Lahtinen*(1) \\ Nanoscience Center, Department of Chemistry, University of Jyväskylä, P.O. Box 35, 40014 Jyväskylä, Finland
}

Supporting Information

ABSTRACT: Controlled synthesis of nanostructure oligomers requires detailed understanding of their wet chemistry and the forces driving the polymerization process. In this paper, we report the main factors affecting the reaction yields of a dithiol-induced synthesis of covalently bound nanocluster dimers and oligomers and present a detailed analysis of possible reaction mechanisms. We synthesize the nanocluster oligomers using monodisperse para-mercaptobenzoic acid ( $p$ MBA)-protected gold nanoclusters with a nominal composition of $\mathrm{Au}_{\sim 250}(p$ $\mathrm{MBA})_{n}$ to minimize ensemble effects on size, shape, and surface structure. Ligand exchange was performed on the nanoclusters with five different dithiol linkers: 5,5'-bis(mercaptomethyl)-2,2'-bipyridine, 4,4"'-thiobisbenzenethiol, benzene-1,4dithiol, 1,4-benzenedimethanethiol, and dimercaptostilbene. Oligomer yields depend strongly on the used dithiol and on the dithiol-to-nanocluster ratio.

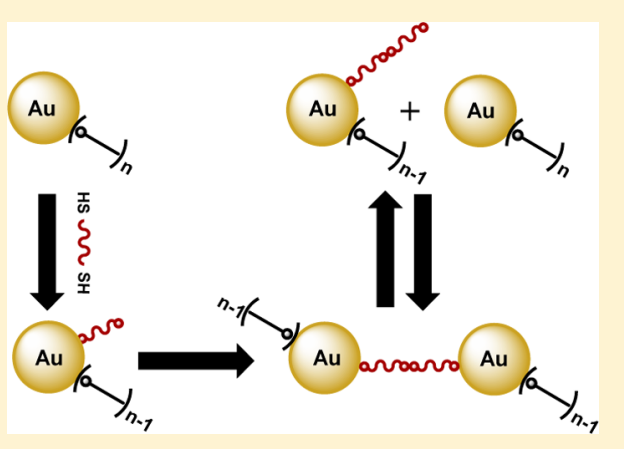
Detailed analysis of the reaction yields in combination with simulations suggests that the system reaches a dynamic equilibrium, where ligand exchange happens continuously forming and breaking nanocluster oligomers that are bound together by short chains of disulfide-bridged dithiols. Despite the dynamic nature of the system, dithiol-induced polymerization of nanoclusters is a general and straightforward approach to produce dimers and larger oligomers of thiol-protected nanoclusters. Our work provides physical insight into, offers tools for, and reveals challenges in the controlled synthesis of covalently bound nanoparticle assemblies.

\section{INTRODUCTION}

Controlled assembly of metal nanoparticles to predetermined superstructures has been under extensive study during the past decades. ${ }^{1}$ Nanoparticle assemblies can have striking optical, ${ }^{2}$ chiro-optical, ${ }^{3}$ and electronic effects, ${ }^{4}$ that can be used, for example, in sensing, ${ }^{5,6}$ photocatalysis, ${ }^{7,8}$ and nanoelectronics. ${ }^{9,10}$ Several approaches have been used to fabricate metal nanoparticle dimers and larger superstructures, such as, simple aggregation, ${ }^{11}$ polymer or silica encasing, ${ }^{12,13}$ templating polymer layers, ${ }^{14}$ lithography, ${ }^{15}$ and wet-chemistry linking approaches. $^{16-21}$ The wet-chemistry linking approaches to synthesize gold nanostructures have the advantage of being scalable to milligram quantities and enable better control of superstructure formation via molecular interactions. For example, DNA-linking and templating has been successfully applied to design well-defined superstructures. ${ }^{9,16}$ Covalent cross-linking of gold nanoparticles with dithiols and multithiols is a well-known approach to form dimers and larger oligomers. ${ }^{17-20}$ However, achieving completely homogeneous samples and control of the interparticle molecular bridges at the level of molecular precision are extremely difficult, and detailed molecular-level understanding of the linking mechanisms has not been fully achieved yet. ${ }^{22}$ This is mainly because colloidal nanoparticles are not homogeneous but form a distribution of sizes, shapes, and surface structures. ${ }^{23}$

Small monolayer-protected gold nanoclusters (AuMPCs) ${ }^{24,25}$ are monodisperse, and they have an atomically well-defined structure, which can be determined by singlecrystal X-ray diffraction ${ }^{26-28}$ or electron tomography. ${ }^{29}$ In contrast to colloidal nanoparticles that form a continuous distribution of sizes, MPCs are known to form a discrete and relatively sparse distribution of preferred cluster sizes. ${ }^{30,31}$ A special class of AuMPCs are thiol-protected AuMPCs, $\mathrm{Au}_{m}(\mathrm{SR})_{n}$, where strong binding and covalent nature of $\mathrm{Au}-$ $S$ bond make them more stable and easier to handle under ambient conditions than commonly used larger nanoparticles. Recently, several thiol-protected AuMPCs with a diameter over $2 \mathrm{~nm}$ have been synthesized. ${ }^{30-32}$ Their properties are approaching the properties of colloidal nanoparticles being metallic and plasmonic while still remaining well-defined and monodisperse. Thanks to their monodispersity and welldefined atomic structure combined with stability and structure-dependent properties, large MPCs and their superstructures are excellent candidates for fundamental studies of nanoscience, for example, for studying emergence of plasmonic effects and hot-electron generation in nanoparticle dimers with unpreceded accuracy.

Several alternatives exist for forming molecular bridges between metal nanoclusters. Cross-linking of small gold clusters with organic molecules has been demonstrated using

Received: March 29, 2018

Revised: May 16, 2018

Published: May 17, 2018 
dithiothreitol, diacetylene, chiral dithiol, diglyme, and biphenyldithiol..$^{20,33-36}$ In our previous work, where we used biphenyl-4,4'-dithiol (BPDT) to cross-link two different MPCs, namely $\mathrm{Au}_{102}(p-\mathrm{MBA})_{44}$ and $\mathrm{Au}_{\sim 250}(p-\mathrm{MBA})_{n}$, we showed that dimers and trimers of monodisperse MPCs can be produced with a straightforward single-step reaction. Here, we extend this work in two ways. We show that the dithiol linking is general in the sense that it works with several different dithiols (in addition to different MPCs, as we demonstrated before ${ }^{36}$ ), and we propose a possible reaction mechanism.

In Experimental Methods section, we briefly review the synthesis that yields dithiol-linked MPC oligomers, and in addition to the commonly used standard analysis methods, we introduce a rapid method to analyze reaction yields of different oligomer fractions quantitatively. In Results and Discussion, we demonstrate that the synthesis is able to produce MPC oligomers with a variety of dithiols. Figure 1 shows the

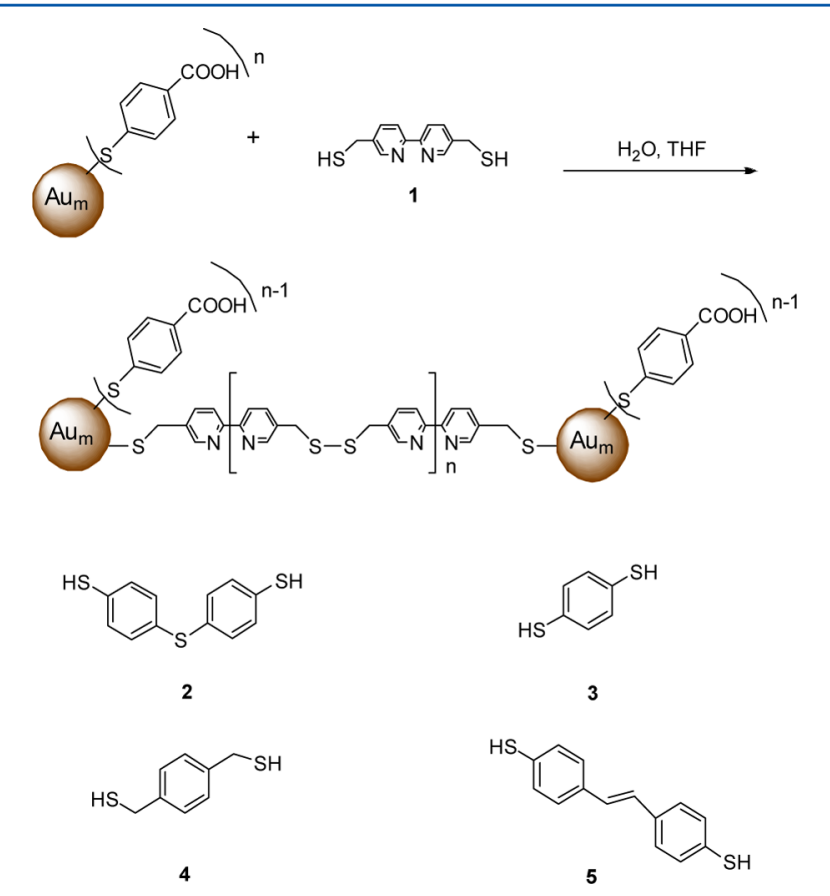

Figure 1. Suggested ligand exchange and the linking reaction of AuMPCs. The scheme also shows the molecular structure of the dithiols used to cross-link $\mathrm{Au}_{\sim 250}\left(p-\mathrm{MBA}_{n}\right)$. BMM-BPy 1, TBBT 2, BDT 3, BDMT 4, and DMS 5.

structures of the used dithiol linkers and the proposed linking mechanism of AuMPCs. We focus on 5,5'-bis(mercaptomethyl)-2,2'-bipyridine (BMM-BPy) 1 and analyze the effects of dithiol-to-MPC ratio and reaction time to oligomer reaction yields. Finally, we discuss the possible reaction mechanisms and narrow down the options with a combination of simulations and experimental data.

\section{EXPERIMENTAL METHODS}

Synthesis of $\mathrm{Au}_{\sim 250}(p-\mathrm{MBA})_{n}$ nanoclusters was carried out, as described in detail in our previous publication. ${ }^{36}$

2.1. Synthesis of Dithiol-Linked $A u_{\sim 250}(p-M B A)_{n}$ Nanocluster Oligomers. Dithiol cross-linked oligomers were prepared by a similar ligand-exchange synthesis previously reported for $4,4^{\prime}$-biphenyldithiol. ${ }^{36}$ Briefly, stock solutions of BMM-BPy 1, 4,4"-thiobisbenzenethiol (TBBT) 2, benzene-1,4dithiol (BDT) 3, 1,4-benzenedimethanethiol (BDMT) 4, and dimercaptostilbene (DMS) $\mathbf{5}$ were initially prepared in tetrahydrofuran. Different dithiol solutions $(\mathbf{1}-\mathbf{5})$ were added to a $\mathrm{Au}_{\sim 250}(p-\mathrm{MBA})_{n}$ water solution with a resulting 2:1 molar ratio (dithiol/MPC). The concentration of $\mathrm{Au}_{\sim 250}(p-\mathrm{MBA})_{n}$ was calculated from the estimated molecular weight of $65 \mathrm{kDa}$ ( $\sim 250$ gold atoms and $\sim 100$ thiolates). The ligand-exchange reaction was quenched after $3.5 \mathrm{~h}$ by adding isopropanol and 5 $\mathrm{M} \mathrm{NH} 4 \mathrm{OAc}$ to the reaction mixture. The resulting black precipitate was centrifuged, and the supernatant containing any remaining free thiol residue was removed. The pellet containing the reaction products was dissolved to $\mathrm{H}_{2} \mathrm{O}$. The linking reaction was carried out for BMM-BPy 1 also with dithiol-toMPC ratios of 1:5, 1:2, 1:1, 2:1, and 10:1. Notable difference to our previously reported synthesis is the omission of adding concentrated $\mathrm{NaOH}$ in the dithiol/MPC reaction mixture. We have discovered that the linking occurs efficiently also without addition of a base.

2.2. Polyacrylamide Gel Electrophoresis. Polyacrylamide gel electrophoresis (PAGE) was used to separate reaction products of $\mathrm{Au}_{\sim 250}(p-\mathrm{MBA})_{n}$ oligomer syntheses with different dithiols. Electrophoretic separation was performed using a BioRad Mini-Protean Tetra System apparatus operated at $130 \mathrm{~V}$. Polyacrylamide gels (15 w/v \%, 29:1 acrylamide/bisacrylamide) with $1.0 \mathrm{~mm}$ thickness were used in the separation. Run buffer in the experiments was $2 \times$ Tris/borate/EDTA.

2.3. UV-Vis Spectroscopy. Optical absorption measurements were carried out with PerkinElmer Lambda $850 \mathrm{UV}$-vis spectrometer with $2 \mathrm{~nm}$ resolution. To measure UV-vis absorption directly from the PAGE gels, each electrophoretically separated column was mechanically cut from the gel and compressed between two quartz microscopy slides. Light beam was accurately positioned on individual gel bands by locally maximizing the absorption using an XYZ-micrometer stage positioned inside the spectrometer.

2.4. Transmission Electron Microscopy. Transmission electron microscopy (TEM) samples were prepared by dropcasting $8 \mu \mathrm{L}$ of dilute deionized water solution of linked nanoclusters on a glow discharged 400 mesh holey carbon copper grid (Ted-Pella ultrathin c). Solution was allowed to deposit for $15 \mathrm{~min}$, after which excess sample was removed, and the grid was allowed to dry under vacuum overnight. Samples were imaged with JEOL JEM-1400HC TEM operated at $80 \mathrm{kV}$, equipped with bottom mounted 11 Megapixel CCD camera (Olympus SIS Quemesa).

2.5. Analysis of Reaction Yields with a Desktop Scanner. We developed a method where a standard desktop scanner is used to evaluate rapidly reaction yields of nanocluster oligomers. First, a desktop scanner (Canon imageRUNNER) was calibrated against a set of neutral density filters, in the following way. Each of the neutral density filters was measured with a UV-vis spectrometer to obtain its absorbance. Then, the same set of filters was scanned with a white A4 paper as a background. The grayscale values obtained from the scanned image were plotted against absorbance of each filter at $500 \mathrm{~nm}$ (see Figure S1). The data were fitted to obtain a conversion curve from grayscale values to the corresponding absorbance values.

The calibration was followed by the actual analysis. First, a normal PAGE run was performed. Then, the wet PAGE gelstill between the glass plates-was immediately scanned. The same white A4 paper that was used in the calibration was used again as the background. The scanned image was converted to a grayscale image. A narrow, rectangular area was selected around 
the darkness maxima of each PAGE column, and the mean intensity of pixels in each row was calculated. The grayscale values were converted to the corresponding absorbance values using the conversion curve obtained from the calibration. The PAGE running distance was calculated by transforming the distance from pixels to millimeters by multiplying with the scanner's known resolution, dots-per-inch. This gives us integrated absorbance as a function of the PAGE running distance in millimeters. Because absorption in the visible range changes negligibly between different MPC oligomer fractions, the measured absorbance is directly proportional to the number of cluster cores in the corresponding gel position. The absorbance values can then be integrated over a band, and the integrated values can be directly converted to reaction yields, as the number of clusters per reaction product, that is, per oligomer in a PAGE band is known from TEM.

2.6. Simulations. Simulations of linking were initialized by taking 100000 nanoclusters, represented as an array of two integers, $n_{\text {cluster }}$ and $n_{\text {dithiol }}$, where initially $n_{\text {cluster }}=1$ and $n_{\text {dithiol }}=$ 0 . Then, dithiols were assigned one-by-one randomly and independently until the desired dithiol-to-MPC ratio was reached, $n_{\text {dithiol }}^{\text {(avg) }}=$ dithiol-to-MPC ratio. Next, a simple MonteCarlo molecular kinetics was performed. At each time-step, a pair of nanoclusters, or later oligomers, was selected randomly, that is, two random array elements, $n^{(i)}$ and $n^{(j)}$, were selected. In the case of direct linking with a single dithiol, a molecular bridge was formed between nanoclusters, if at least one of the two nanoclusters had dithiol available for linking, $n_{\text {avail }}^{(i)}>0$ or $n_{\text {avail }}^{(j)}>0$. In the case of linking via disulfide bridge, a molecular bridge was formed between nanoclusters, if both nanoclusters had at least one dithiol available for linking, $n_{\text {avail }}^{(i)}>0$ and $n_{\text {avail }}^{(j)}>$ 0 . Whether any dithiols were available for linking or not was determined by how many nanoclusters and dithiols were already in the nanocluster or oligomer. In the case of direct linking with a single dithiol, each molecular bridge reduced available dithiols for linking by one, $n_{\text {avail }}=n_{\text {dithiol }}-\left(n_{\text {cluster }}-\right.$ $1)$. In the case of linking via disulfide bridge, each molecular bridge reduced available dithiols for linking by two, $n_{\text {avail }}=$ $n_{\text {dithiol }}-2\left(n_{\text {cluster }}-1\right)$. If enough available dithiols were found, a new molecular bridge was created and the selected pair of nanoclusters/oligomers was merged as a new larger oligomer, $n^{(k)}=n^{(i)}+n^{(j)}$, and the old ones were removed, $n^{(i)}=n^{(j)}=0$. This procedure was repeated until the distribution of oligomers converged.

Breaking of dithiol bridges, breaking via ligand exchange, formation of dithiol polymers, passivation of dithiols with $p$ $\mathrm{MBA}$, unequal ligand-exchange probabilities, and so forth were not considered in the simulations to keep the model minimal. Addition of these effects would introduce adjustable parameters complicating the interpretation and discussion of simulation results without adding significant value.

\section{RESULTS AND DISCUSSION}

3.1. Qualitative Analysis of $A u_{\sim 250}(p-M B A)_{n}$ Oligomers. Crude products from the $\mathrm{Au}_{\sim 250}(p-\mathrm{MBA})_{n}$ ligand-exchange synthesis with different dithiols were analyzed initially by PAGE. Reaction products of all five tested dithiols $(\mathbf{1}-\mathbf{5})$ produced qualitatively similar patterns, as seen in Figure 2. In all cases, distinct bands formed above the unlinked cluster band, which means that reaction products in these bands have lower mobility than the reference unlinked cluster. Similar patterns were observed also in our previous study, where BPDT was used as the linker molecule. ${ }^{36}$

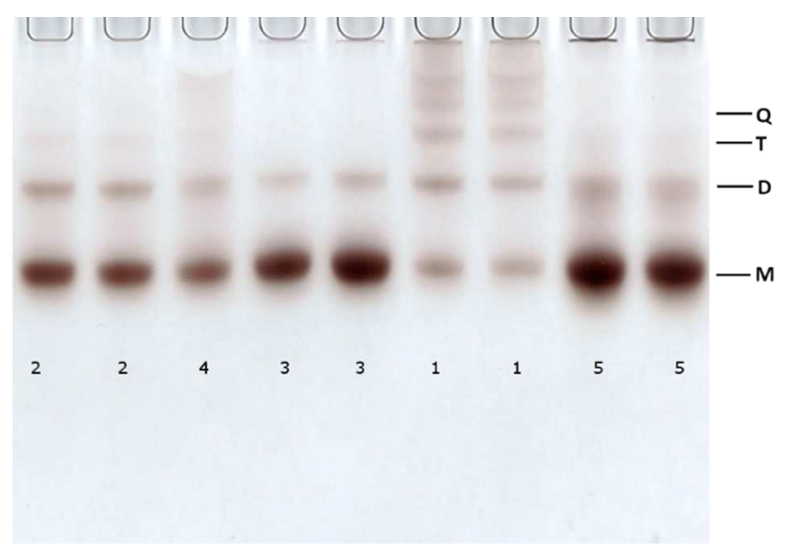

Figure 2. PAGE gel showing bands of $\mathrm{Au}_{\sim 250}(p-\mathrm{MBA})_{n}$ linked with different dithiols. The index of the dithiol used is specified below each column (see Figure 1). Ratio of dithiol-to-MPC was 2:1 in all cases.

We confirmed that the observed bands originate from different oligomers using TEM imaging, as shown in Figures 3

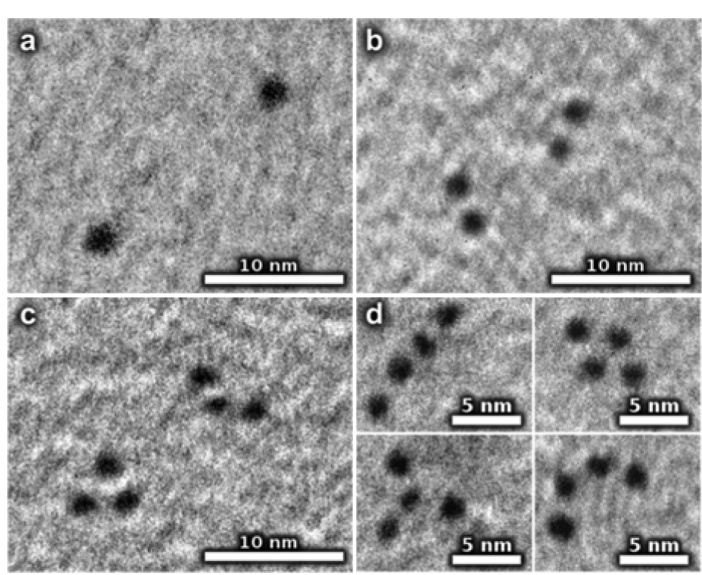

Figure 3. TEM micrographs showing (a) monomers of $\mathrm{Au}_{\sim 250}(p$ MBA $)_{n}$ from $\mathrm{M}$ band; (b) dimers linked with BMM-BPy 1 from D band; (c) trimers from $\mathrm{T}$ band; and (d) tetramers from $\mathrm{Q}$ band.

and S2-S5, for BMM-BPy 1 . The most mobile $\mathrm{M}$ band consists of monomers, whereas D-, T-, and Q bands contain a dominant fraction of dimeric, trimeric, and tetrameric forms, respectively. As all of the tested linking reactions with different dithiols produced PAGE patterns indicating significant presence of oligomers, the dithiol linking approach appears to be a general synthetic strategy to produce covalently bound nanocluster oligomers.

3.2. Quantitative Analysis of $A u_{\sim 250}(p-M B A)_{n}$ Oligomers. Although similar PAGE patterns are observed with all dithiols, the reaction yields of different oligomer fractions depend strongly on the particular dithiol used. To compare the relative yields between dithiols, we determined them quantitatively. We applied a rapid method inspired by the densiometric analysis of Western blot ${ }^{37}$ and the use of PAGE to separate different MPCs. ${ }^{30,38,39}$ The method uses a standard desktop scanner to analyze a complete PAGE gel at once. Each PAGE gel has multiple wells, each of which can be used to separate products of a different reaction. Single PAGE run followed by scanning can therefore analyze reaction yields of ten or more MPC linking reactions at once (see Experimental Methods section for the details of the method). 
Normalized absorbance profiles obtained with the scanning method along each column of PAGE bands of Figure 2 are shown in Figure 4. To validate our scanning method, we

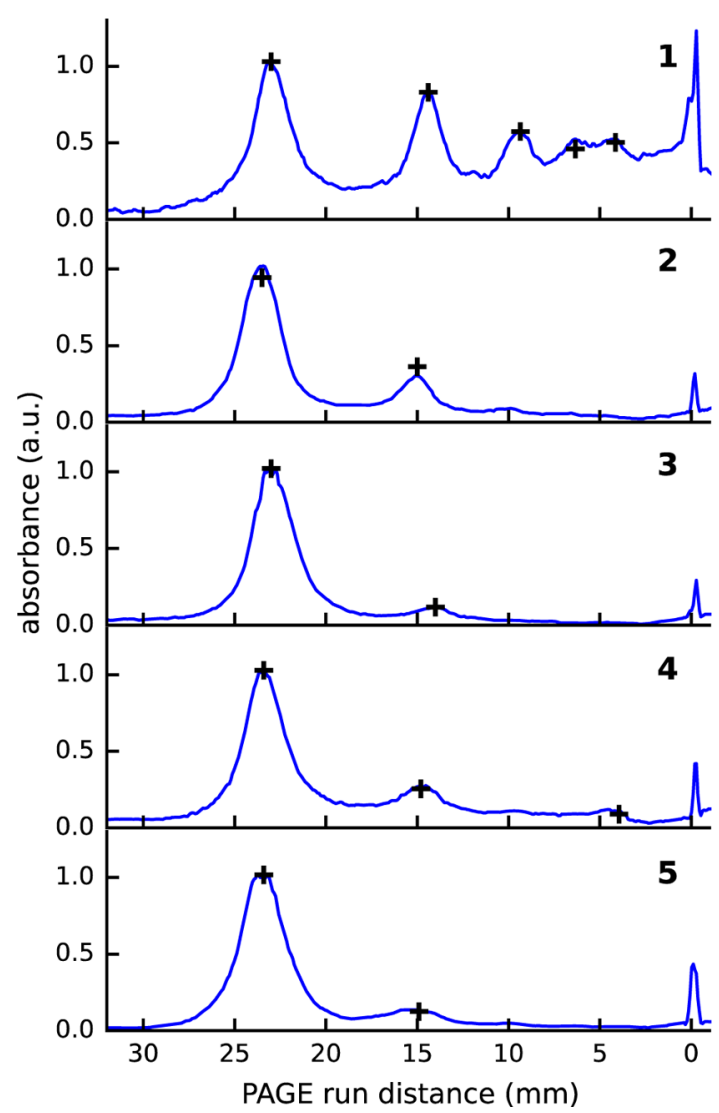

Figure 4. Absorbance of linked $\mathrm{Au}_{\sim 250}(p-\mathrm{MBA})_{n}$ as a function of the PAGE running distance for different dithiols. Indices of the dithiols are given in the top-right corner of each panel. The solid blue lines are the integrated absorbances obtained with the scanning method along each column of the PAGE gel. The black crosses are the integrated absorbances obtained from the UV-vis spectrum and scaled for an optimal fit to the scanning method.

performed a UV-vis spectroscopy measurement for each visible band in the same PAGE gel. The absorption spectrum (see Figure S6) was integrated from 400 to $600 \mathrm{~nm}$ to obtain an integrated absorbance. The integrated absorbance is directly proportional to the local concentration of nanoclusters because the UV-vis spectrum changes negligibly in this region between different oligomers. The integrated absorbance values obtained with this method are shown in Figure 4 as crosses.

The results of the scanning method for BMM-Bpy 1, BDT 3, BDMT 4, and 4,4'-DMS 5 linked $\mathrm{Au}_{\sim 250}(p \text {-MBA })_{n}$ oligomers are in near-perfect agreement with the results determined from the integrated UV-vis absorbance, as seen in Figure 4. The relative absorbance of TBBT 2 differs somewhat between the two methods, although the trend is similar. We speculate that this small discrepancy originates from experimental uncertainties in acquiring UV-vis spectra from the cut gel samples. The scanning approach is significantly faster and easier to measure than the UV-vis approach, and therefore it provides a fast and reliable way to obtain local nanocluster concentration profiles along each PAGE column for linked MPC systems.

3.2.1. Linking Efficiency. The profiles in Figure 4 show that BMM-BPy $\mathbf{1}$ is clearly the most effective in forming higher order oligomers when the dithiol-to-MPC molar ratio was 2:1, whereas BDT 3 is the least effective. It is not surprising that BMM-BPy 1 is more efficient than BDT because BMM-BPy 1 is significantly longer than BDT 3 . Actually, BDT $\mathbf{3}$ is so short, sulfur-to-sulfur distance being $0.6 \mathrm{~nm}$, that it would probably require at least three BDT 3 molecules to bridge metallic cores of two MPCs through their $p$-MBA ligand layers, if the thickness of the ligand layer is evaluated from the known structure of $\mathrm{Au}_{102}(p-\mathrm{MBA})_{44 \cdot{ }^{28}}$ However, the length of the dithiol linker cannot be the only factor affecting the efficiency of oligomer formation. 4,4'-DMS 5 has practically the same length $(1.3 \mathrm{~nm})$ as BMM-BPy $\mathbf{1}(1.2 \mathrm{~nm})$, but its efficiency for producing dimers is an order of magnitude lower than BMMBPy 1 and only just above the shortest linker BDT 3 (see Figure 4). This clearly indicates that also other factors than the length of the linker are important to oligomer formation. Possible candidates are, for example, steric effects and dithiol$p$-MBA interactions. One would also expect that the chemical group attached to the terminal sulfurs would play an important role in the reaction. This is likely to be the case, but again, it cannot be the only determining factor. For example, BMM-BPy 1 and BDMT 4 have the same methylphenyl group attached to the sulfur but still have significantly different linking efficiencies. Therefore, the linking efficiency must be an interplay between the different factors mentioned above, and further studies are required to explain different contributions in detail. Our findings are in agreement with Salassa et al., who demonstrated that ligand distributions of $\mathrm{Au}_{25}(\mathrm{SR})_{18-x}\left(\mathrm{SR}^{\prime}\right)_{x}{ }^{-}$depend on the thiol pair used for the synthesis and for the subsequent ligand exchange. $^{40}$

3.2.2. Reaction Conditions. Next, we will focus on the most efficient linker molecule BMM-BPy 1 and analyze its reaction yields in different reaction conditions. We performed the ligand exchange of $\mathrm{Au}_{\sim 250}(p-\mathrm{MBA})_{n}$ with BMM-BPy 1 using different dithiol-to-MPC molar ratios of 1:5, 1:2, 1:1, 2:1, and 10:1. PAGE gel separation of the first four ratios are shown in Figure $S 7$, and the corresponding absorption profiles are shown in Figure 5. A PAGE gel including ratio 10:1 is shown in Figure S8a. The absorption profiles show that the lowest dithiol concentration (1:5) strongly favors monomeric form yielding only a small fraction of dimers and negligible amounts of higher oligomers, as expected (see Figures S8b and S9). The trimer and larger oligomer yields increase rapidly when dithiol-tonanocluster molar ratio is increased from $1: 5$ to $1: 2,1: 1$, and $2: 1$. As the concentration is increased further, the reaction begins to produce large amounts of nanocluster aggregates that remain immobile in the PAGE wells, as seen in Figure S8.

A similar trend can be observed in TEM statistics where relative abundances of different oligomers in the crude product were determined from TEM micrographs. The statistics of two different linker-to-MPC molar ratios are shown in Figure S9. The fractions of dimers, trimers, and larger oligomers are significantly higher in 2:1 molar ratio than in 1:5 ratio. PAGE and TEM distributions are not entirely comparable because some noncovalent agglomeration is prone to happen in TEM sample preparation, nevertheless, the trend is clear.

To gain more insights to the reaction mechanism, we performed quantitative analysis of reaction yields as a function of the dithiol-to-MPC ratio by processing the absorbance data produced by the scanning approach in a following way. The reaction yields were estimated from the absorbance data by integrating the area under each PAGE band. The integrated area is proportional to the number of MPCs in the band. As 


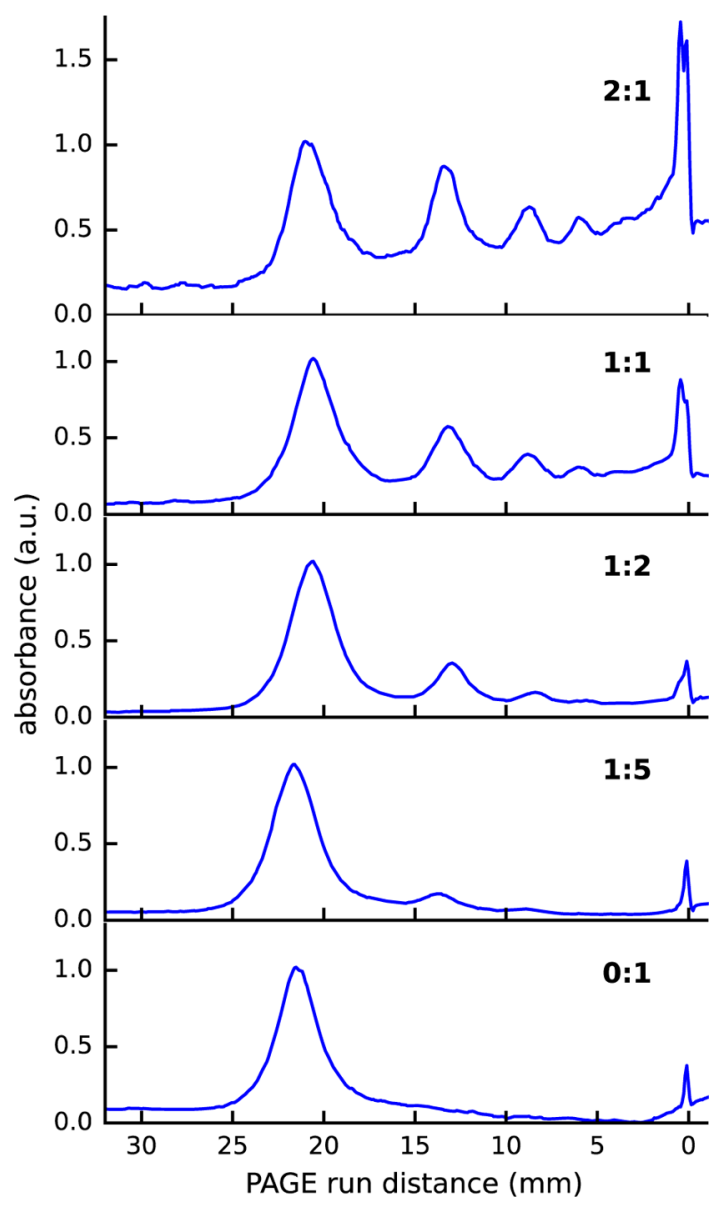

Figure 5. Absorbance of BMM-BPy 1-linked $\mathrm{Au}_{\sim 250}(p-\mathrm{MBA})_{n}$ as a function of the PAGE running distance. The molar ratios are given in the top-right corner of each panel. The lowest panel shows the unlinked $\mathrm{Au}_{\sim 250}(p-\mathrm{MBA})_{n}$ reference band (ratio 0:1).

each band has approximately a Gaussian profile, we fitted a set of Gaussian functions on top of a linear background (see Figure S10). The number of MPCs in a band can be converted to its relative concentration of oligomers (relative to other bands) by dividing the number of MPCs in the band by the number of MPC per oligomer known from TEM. The oligomer reaction yields as a function of dithiol-to-MPC ratio for $\mathrm{Au}_{\sim 250}(p$ MBA) ${ }_{n}$ linked with BMM-BPy 1 are shown in Figure 6a.

3.2.3. Saturation of Oligomer Yields. The reaction yields decrease approximately exponentially with respect to the number of nanoclusters in the oligomer, that is, monomer-todimer ratio is close to dimer-to-trimer ratio, and trimer-totetramer ratio. A similar behavior is observed in step-growth polymerization, ${ }^{41}$ which has been demonstrated to happen also for nanoparticles. ${ }^{42}$ In step-growth polymerization, bifunctional (or multifunctional) monomers form dimers with probability (1 $-p(t)) p(t)$, and longer oligomers with

$$
P_{n}=(1-p) p^{n-1}
$$

where $P_{n}$ is probability of finding an oligomer with $n$ monomeric units, and $p$ is the probability for a single polymerization step. The exponential curves are shown as solid lines in Figure 6a, ratio-versus-yield-a correspond to $P_{n} /$ $P_{1}$. The above equation (eq 1) holds only for ideal bifunctional monomers $(-\mathrm{A}-)$ or bifunctional polymerizing units (e.g., $-[\mathrm{A}-\mathrm{B}]-)$ and only for the equimolar case. For a non-
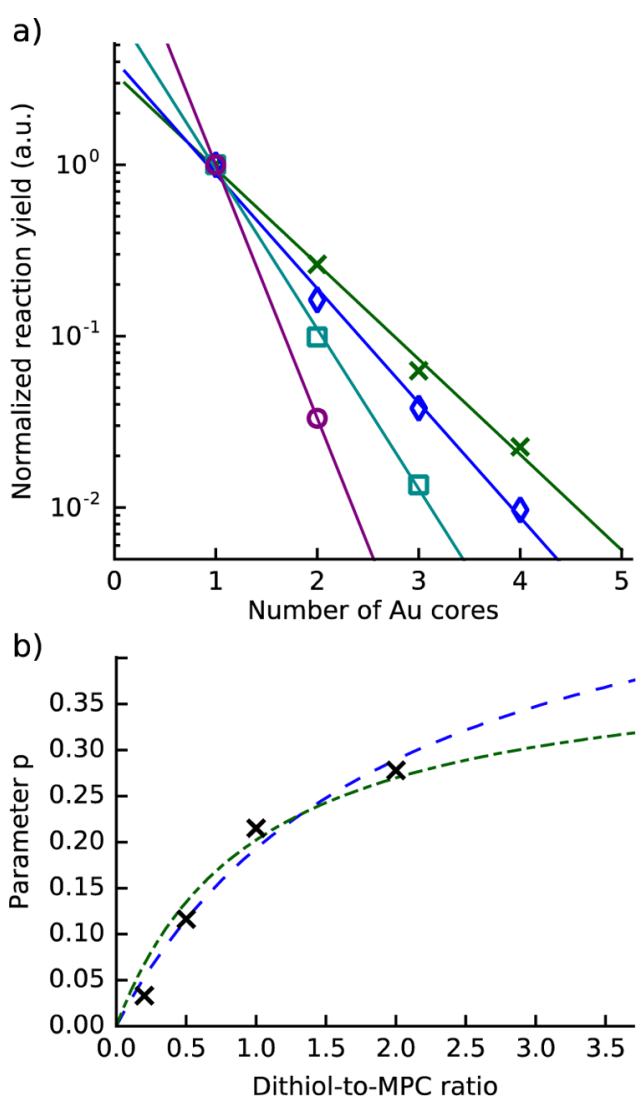

Figure 6. (a) Oligomer reaction yields normalized to the monomer yield as a function of the oligomer size for dithiol-to-MPC ratios of 1:5 (purple circles), 1:2 (cyan squares), 1:1 (blue diamonds), and 2:1 (green crosses). The reaction yields decrease approximately exponentially as the number of nanoclusters in the oligomer increases. Solid lines are linear least-squares fits to $\log _{10}\left(P_{n} / P_{1}\right)$. (b) Fit parameter $p$ as a function of dithiol-to-MPC ratio (black crosses), fit to eq 4 with $r_{\mathrm{AB}}$ equal to dithiol-to-MPC ratio $R$, that is, one dithiol per MPC (dot-dashed green), fit to eq 4 with $r_{\mathrm{AB}}=R / 2$, that is, two dithiols per MPC (dashed blue).

equimolar case, we can take into account the dependence on the dithiol-to-MPC ratio by replacing the degree of polymerization for the equimolar case

$$
X=\left(1-p_{\text {eq }}\right)^{-1}
$$

by the degree of polymerization for a nonequimolar case

$$
X=\left(1-p_{\mathrm{eq}} \frac{2}{1+r_{\mathrm{AB}}^{-1}}\right)^{-1}
$$

where $r_{\mathrm{AB}}$ is the ratio of $\mathrm{A}$ and $\mathrm{B}$ in the bifunctional unit. ${ }^{41}$ From these equations, we see that the fit parameter $p$ should depend on the ratio $r_{\mathrm{AB}}$ as

$$
p\left(r_{\mathrm{AB}}\right)=p_{\mathrm{eq}} \frac{2}{1+r_{\mathrm{AB}}^{-1}}
$$

In Figure $6 \mathrm{~b}$, the fit parameter $p$ is shown as a function of the dithiol-to-MPC ratio $R$ together with fits to eq 4 with $r_{\mathrm{AB}}=R$ and $r_{\mathrm{AB}}=R / 2$. The first fit, $r_{\mathrm{AB}}=R / 2$, corresponds to a polymerization unit with one dithiol per $\mathrm{MPC}$, that is, one dithiol directly connecting two MPCs (-D-MPC-DMPC-). Whereas the second fit, $r_{\mathrm{AB}}=R / 2$, corresponds to two dithiols per MPC, that is, two dithiols connecting two 
MPCs via a disulfide bridge (-D-MPC-D-D-MPC-D-). Experimental observations and both fits have the same trend, where the polymerization probability $p(R)$ changes rapidly at low dithiol-to-MPC ratios, but become saturated at higher ratios. In other words, increasing the dithiol-to-MPC ratio beyond certain point does not increase the yields of dimers, trimers, and tetramers significantly, but leads to increasing formation of large aggregates. The fits are used here only to demonstrate the trend. We will present a more rigorous analysis and an elaborate discussion below.

3.2.4. Reaction Time and Equilibrium. A typical way to control a polymerization reaction is to control its reaction time. We examined this aspect by performing syntheses using 0.25 , $0.5,3.5$, and $7 \mathrm{~h}$ reaction times for the equimolar case (i.e., 1:1 ratio). The resulting oligomer yields are shown in Figure 7 ,

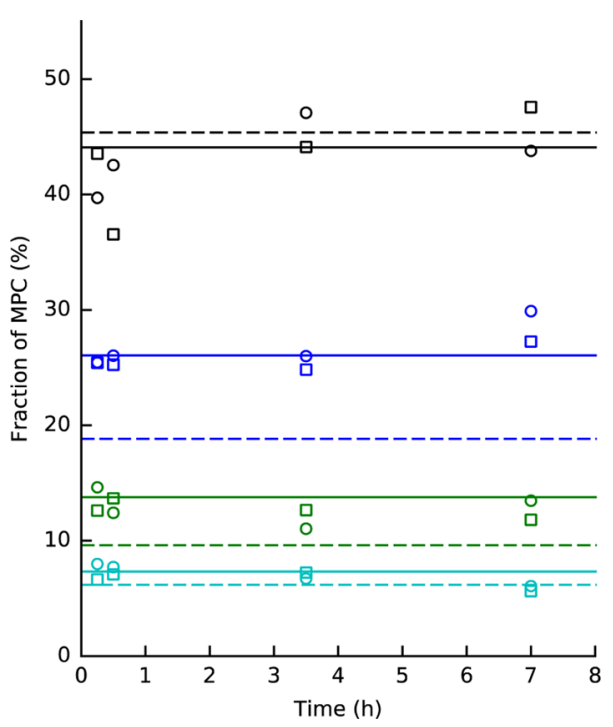

Figure 7. Oligomer yields for equimolar case $(1: 1)$ as a function of the reaction time $(0.25,0.5,3.5$, and $7 \mathrm{~h})$ of two different syntheses. The shapes (circles and squares) denote different nanocluster synthesis batches. At each time, we have four values corresponding with (from top to bottom) monomer (black), dimer (blue), trimer (green), and tetramer (cyan). Simulated oligomer yields at equilibrium for ligandexchange linking (solid) and for disulfide linking (dashed) with the optimal linking activities of 35 and $80 \%$, correspondingly, are shown as vertical lines (for details, see discussion below). The reaction yields are given as a fraction of MPCs in the corresponding oligomeric form. The linking activity (\%) is defined as the fraction of dithiols that act as molecular bridges, with respect to the total number of the dithiols.

where we observe that the reaction yields have no significant dependence on time. Therefore, the reaction must have reached its end or equilibrium, or at least, be in the slowly varying asymptotic region near the equilibrium. We will use this information in the following discussion of full kinetic model.

3.3. Kinetic Model. We have shown that our experimental results agree surprisingly well with step-growth polymerization, and it serves as a good starting point for the discussion, but it is an oversimplification of the process. In reality, the ligandexchange process must be (at least up to a certain degree, for example, within preferred sites) a stochastic process, where ligand exchange can happen once, twice, or more times per MPC. This leads to MPCs with one, two, or more dithiol "arms", whereas some of the MPCs are left without any dithiol arms. The distribution of arms per MPC depends on the dithiol-to-MPC ratio. The ligand exchange can also happen in reverse direction, where an already exchanged dithiol is replaced by an exchanged $p$-MBA from the solution. In addition, we have another competing process: the disulfide bridge formation between dithiols.

Therefore, the key factors in the formation of the equilibrium (or a long-lived metastable state) in dithiol-MPC systems are (1) ligand-exchange rate, (2) disulfide formation rate, (3) ligand-exchange equilibrium between $p$-MBA and dithiol, and (4) disulfide equilibrium. Some of the rates and equilibriums might be different for free versus MPC-bound dithiols, and for monomers versus oligomers. To gain insights into this rather complex system, we will test different simplifying hypotheses and their validity. We have collected different hypotheses and their results in Figures 8 and S11.

3.3.1. Hypothesis: Fast Disulfide Formation (Figure S11X). Let us first assume that disulfide formation reaction strongly favors disulfides over thiols/thiolates. This assumption is supported by earlier research where dithiols were observed to polymerize in water-forming disulfide-bridged chains and rings. ${ }^{43,44}$ In addition, our observation that when we add dithiol to water alone, we observe the formation of a white milky haze indicating the same polymerization process.

If we combine a hypothesis of irreversible disulfide formation with a hypothesis that this reaction is much faster than ligand exchange, we should observe long polymer chains of dithiols connecting two MPCs at the ends (see Figure S11). This would result in extremely low oligomer yields at equimolar dithiol-toMPC ratio, however, as we observe significant amounts of oligomers, for example, in Figure 7, this combination of hypotheses is not valid.

3.3.2. Hypothesis: Fast Ligand-Exchange (Figure S11a). If we take an opposite hypothesis for the reaction speeds, that is, if ligand-exchange reaction is much faster than disulphide formation, a metastable state begins to form, where MPCs have one or more ligands exchanged to dithiols, but no molecular bridges have formed between MPCs yet (see Figures 8a and S11a). How many of the dithiols become exchanged depends on the dithiol, for example, some dithiols will have strong steric hindrance or have chemical groups that strongly repel neighboring $p$-MBAs leading to low-exchange probabilities, whereas others might be preferred over $p$-MBA for the opposite reasons. Nevertheless, in general, there exists a strong entropic force to drive dithiols from the solution to the ligand layer at equimolar dithiol-to-MPC ratio for the following reason. Initially, all dithiols are in the solution and all $p$-MBAs are in the ligand layer. Entropy drives dithiols to the ligand layer until the ligand layer and the solution have equal dithiol-to- $p$-MBA ratio $(\sim 1: 100$ in the equimolar case). Because the ligand layer has two orders of more thiols than in the solution, entropy drives dithiols to the ligand layer, until it has two orders of more dithiols than in the solution. In other words, unless dithiols are energetically disfavored, the metastable state has nearly all dithiols bound to MPCs.

3.3.2.1. Simplifying Assumption: Entropy Dominates. We assume, as given below, that the ligand exchange does not energetically favor or disfavor dithiol, and that the equilibrium is determined mainly by entropy. If dithiol is favored or disfavored slightly, this leads to readjustment of distribution without altering the qualitative behavior of the system.

3.3.2.2. Simplifying Assumption: Negligible DithiolDithiol Interactions. As more than one dithiol per MPC must be exchanged to form trimers and larger oligomers, we must consider dithiol-dithiol interactions. We will assume that 


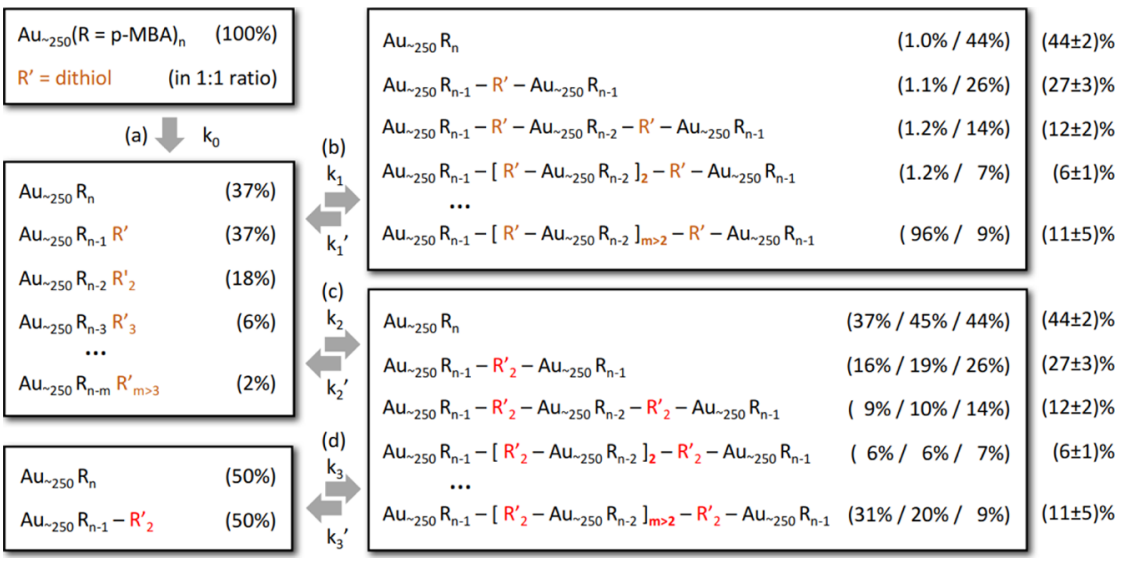

Figure 8. Schematic presentation of hypotheses and their outcomes (see also Figure S11): (a) formation of a metastable state if ligand exchange is faster than disulfide formation, (b) formation of oligomers bridged by single dithiol, (c) formation of oligomers bridged by two dithiols with a disulfide bond between them, (d) re-equilibration caused by ligand exchange after the initial formation of oligomers via disulfide bridging. Values in parenthesis are the fractions of MPCs in the specified form. Values in the top-right panel are for 100\% (left) and for 35\% (right) linking activity. Values in the bottom-right panel are for $100 \%$ (left) and for $80 \%$ (center) linking activity without re-equilibration, and for $70 \%$ linking activity after the re-equilibration via ligand exchange. Values in the parentheses outside of the boxes are the average of the experimental values for $3.5 \mathrm{~h}$ and $7 \mathrm{~h}$ reaction times, as shown in Figure 7. The error is calculated as the maximum absolute deviation from the average.

only a few dithiols exchange, and that the exchanged dithiols are on average far enough from each other to not interact significantly. If no significant dithiol-to-dithiol interaction exists when dithiols are bound to MPC, then the ligand-exchange process should be random and independent from how many dithiols have already been exchanged. As we are using low dithiol-to-MPC ratio, the assumption of negligible dithioldithiol interactions is justified.

3.3.2.3. Metastable State. Examples of simulated metastable states based on the above assumptions of fast, nearly one-way ligand-exchange reaction, where exchanges happen randomly and independently, are shown in Figure S12. When dithiol-toMPC ratio is below 1:10, fraction of MPCs with one dithiol grows linearly as a function of ratio and practically no MPCs with two or more dithiols exist. Behavior diverges rapidly from linear as the ratio grows, and at equimolar ratio, only $37 \%$ of MPC have one dithiol and still $37 \%$ of MPC have no dithiol, $18 \%$ have two dithiols, $6 \%$ have three, and the remaining $2 \%$ have four or more dithiols. Above equimolar ratio, the fraction of MPCs with multiple dithiols increases rapidly, and at 4:1 ratio, it reaches $90 \%$.

After the formation of the metastable state, molecular bridges begin to form between MPCs. This could happen in two ways. The first option is that a single dithiol forms a direct bridge from one MPC to another (see Figures $8 \mathrm{~b}$ and S11b). The second option is that two or more dithiols form disulfide bridges that connect two MPCs (see Figures $8 \mathrm{c}$ and S11c). Simulations of these two cases are shown in Figure 9, where we have still assumed that both reactions are one way $\left(k_{1}^{\prime}=k_{2}^{\prime}=0\right)$.

3.3.3. Hypothesis: Direct Linking with Single Dithiol (Figure S11b). We observe that direct linking with a single dithiol would produce practically only large aggregates at equimolar ratio (see Figures $8 \mathrm{~b}$ and $\mathrm{S} 11 \mathrm{~b}$ ), which does not match with the experiment (see, e.g., Figure 7). Either direct dithiol linking does not happen or all dithiols are not active in linking. If we assume that only $35 \%$ of dithiols form dithiol bridges between MPCs, that is, the effective dithiol-to-MPC ratio is 0.35 , we have an excellent match with the experimental reaction yields (see Figure 7 ). Here, we defined the linking activity (\%) as the fraction of the dithiols that act as molecular

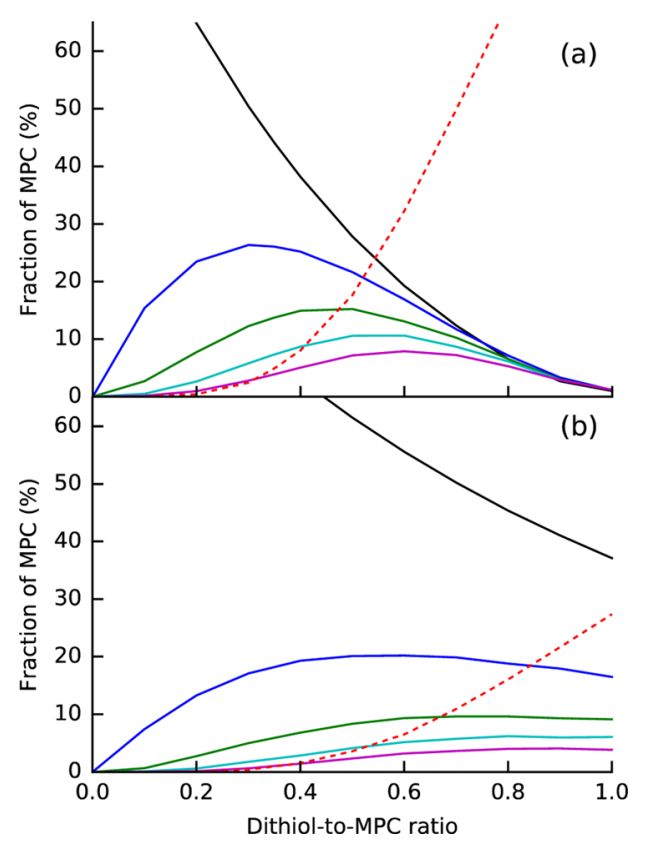

Figure 9. Simulated fractions of MPCs in the form of monomers (black), dimers (blue), trimers (green), tetramers (cyan), pentamers (magenta), and larger than pentamer (dashed red) for (a) linking where a molecular bridge between MPCs forms via ligand exchange, and for (b) linking where a bridge forms via disulfide bond formation. Panel (a) was simulated with one dithiol molecule in the bridge (minimum for a dithiol bridge), and panel (b) with one dithiol pair (minimum for a disulfide bridge).

bridges, with respect to the total number of the dithiols in the system that includes free dithiols and exchanged but nonbridging dithiols, in addition to the bridging ligands. Assuming reduced "linking activity" of $35 \%$ for dithiols is reasonable because it is possible that (1) not all dithiols become exchanged, that (2) not all exchanged dithiols form bridges, and/or that (3) exchanged $p$-MBAs passivate some of the dithiols.

3.3.4. Hypothesis: Indirect Linking via Disulfide Bridge (Figure S11C). Direct linking with a single dithiol and linking 
activity of $35 \%$ offers a one-possible explanation, but other explanations are not yet ruled out. The second option for forming oligomers starting from the metastable state is that the exchanged dithiols form disulfide bridges between the MPCs (see Figures $8 \mathrm{c}$ and S11c). If we assume that this is an irreversible reaction and that ligand exchange does not happen anymore $\left(k_{2}>0 ; k_{1}, k_{1}^{\prime}, k_{3}^{\prime}, k_{3}^{\prime}, k_{2}^{\prime}=0\right)$, our simulations at equimolar ratio yield a distribution of oligomers that is somewhat similar to the experimental data. The match is much better than direct linking with $100 \%$ linking activity, but it is clearly not as good as direct linking with $35 \%$ activity. Even if we take an optimal linking activity of $80 \%$ for the disulfide linking, the match is not nearly as good as the direct linking with the optimal activity of $35 \%$, especially for dimer (see Figure 7).

The above assumption that ligand exchange does not happen after the metastable state has formed was made to simplify simulations and discussion, and it is clearly not valid in a general case. The only case when it would be valid is when dithiols are energetically strongly favored over $p$-MBAs in the ligand layer. Therefore, we consider next a more general case, where the ligand-exchange process is a continuous, two-way process that reaches a dynamic equilibrium.

3.3.5. Hypothesis: Ligand-Exchange Equilibrium Together with Slow Disulfide Formation (Figure S11d). If we make an assumption that the ligand-exchange process is happening to both directions continuously and that the already formed disulfide bridges do not break, we have the following process: random, two-way dithiol exchange $\left(k_{2}, k_{2}^{\prime}, k_{3}, k_{3}^{\prime},>0\right)$ together with slow, one-way disulfide bridge formation $\left(k_{1}>0 ; k_{1}^{\prime}=0\right.$; $\left.k_{2}, k_{3} \gg k_{1}\right)$. Initially, the metastable state forms reaching dynamic equilibrium as before. Occasionally, disulfide forms bridging two MPCs together with a chain of two dithiols to form a dimer. As the ligand exchange is an ongoing process, the formed disulfide-bound dithiol chain can also be exchanged. This results in MPC without dithiols (more precisely, MPC that has lost one of its dithiol chains) and MPC with a chain of dithiols that is bound only from one end. MPC, with a chain bound only from one end, can bind to any other MPC without forming a new disulfide bridge. In other words, it behaves similar to MPC in the direct dithiol linking via ligand exchange, but instead of a single dithiol, it has a chain of dithiols attached to it. Slowly, every dithiol becomes part of a disulfide bridge, and in the end, the ligand-exchange process happens only with disulfide-bridged chains (see Figures $8 \mathrm{~d}$ and S11d).

3.3.5.1. Chains of Two Dithiols. Let us ignore the formation of dithiol chains longer than two for a moment. The above process will end up to an equilibrium that is equal to the direct ligand-exchange linking with a single dithiol, except that a chain of two bridged disulphides connect MPCs instead of a single dithiol, that is, the chain acts as a single, long dithiol. The dithiol-to-MPC ratio of the direct linking case must be now replaced by chain-to-MPC ratio, but otherwise the two processes lead to exactly the same distribution.

Because a chain of two dithiols has twice as many dithiols as a single dithiol, the optimal dithiol linking activity is simply $70 \%$, and the match with the experiment is as good as with a single dithiol and 35\% linking activity.

3.3.5.2. Distribution of Chain Lengths. When chains longer than two dithiols form, the linking activity for the chain rises above $70 \%$. The upper limit of $100 \%$ is reached, when the average chain length is approximately 2.85 dithiols, for example, with an exponential distribution, where a chain of two dithiols has a probability of $54 \%$, a chain of three $25 \%$, a chain of four $11 \%$, and so forth. This is relevant as we have previously demonstrated that BPDT, a dithiol of similar size as BMM-BPy 1, forms dimers that have chains of three or more dithiols, in addition to chains of two. ${ }^{36}$

3.3.5.3. Dynamic Chains. Formation of chains longer than two dithiols is actually quite likely in the above scenario (see Figures $8 \mathrm{~d}$ and S11d). Chains can grow or shrink via thioldisulfide exchange, ${ }^{45}$ as long as free thiol is present in the solution. Nevertheless, thiol-disulfide exchange reaction should have lower rate than ligand exchange, or otherwise, the distribution of oligomer yields would resemble more the dynamics of the disulfide linking without ligand exchange (see Figures 8c and S11c).

3.3.6. Summary of the Kinetic Model. After this discussion, we can summarize the requirements for reproducing the experimental data for BMM-BPy 1: (1) equilibrium has been reached, (2) equilibrium has significant fraction of dithiols bound to MPCs, (3) equilibrium favors disulfide formation over breaking $\left(k_{2}>k_{2}^{\prime}\right)$, and (4) ligand exchange happens more frequently than disulfide formation $\left(k_{1}\right.$ or $\left.k_{3} \gg k_{2}\right)$. Requirements 1 and 2 are supported directly by experimental observations. Requirement 3 is supported directly by experimental data for short chains of dithiols (and it is irrelevant for the unlikely case that linking happens only via single dithiols). Requirement 4 is supported by a combination of simulations and experiments. These requirements lead to a distribution of oligomers that originates (dominantly) from a process, where molecular bridges between MPCs form via ligand exchange. The simulated distribution reproduces the experimental data extremely well, when the average chain length for the molecular bridges is from a single dithiol up to 2.85 dithiols and the corresponding dithiol linking activity is from $35 \%$ up to $100 \%$, respectively.

\section{CONCLUSIONS}

We have demonstrated that covalent linking between MPCs can be achieved with several different dithiols indicating generality of the ligand-exchange-based dithiol-linking approach. We observed that oligomer yields depend strongly on the dithiol used, but we found no simple trend based on the length of the dithiol or the functional groups in the dithiol. The yields depend strongly also on the dithiol-to-MPC ratio. Too low ratio leads to low oligomer yields, whereas too high ratio leads to excessive aggregation that reduces the yields. The optimal ratio is found in a relatively narrow range, for BMMBPy 1, between 1:1 and 2:1. Nevertheless, we showed that this range can be found in a straightforward way by a set of syntheses with different ratios followed by a PAGE run and scanning of the PAGE gel with a desktop scanner. The scanning approach is also suitable for extensive studies with different dithiols, MPCs, and reaction conditions to find trends and to gain deeper understanding of the dithiol-induced oligomerization of MPCs and nanoparticles.

We discussed several different options for a possible reaction mechanism based on the experimental observations obtained with our rapid scanning approach and simulations of reaction kinetics. We found that the best match with the experimental observations is achieved with simulations of a system at dynamic equilibrium, where MPCs are linked by short chains of disulfide-bridged dithiols that continuously form and break via a ligand-exchange process. The shortest possible chain length is one dithiol requiring that only $\sim 35 \%$ of the dithiols are 
contributing to the molecular bridges between MPCs, in the case of BMM-BPy 1. However, based on our previous results on a similarly sized BPDT dithiol, the dithiol chains are more likely two or more dithiols long, the upper limit for the average chain length being 2.85 dithiols, in the case of BMM-BPy 1 .

Our result that the system at dynamic equilibrium, where ligand exchange happens continuously, is in agreement with the recent studies of ligand exchange in MPCs. ${ }^{40,46,47}$ This has implications for the stability of the system. As long as dithiol-toMPC ratio remains unchanged and the distribution of oligomers is not altered in other ways, the system should be stable and self-repairing in solution. However, immediately, if the equilibrium distribution is disturbed, for example, by extracting one pure fraction of dimers, the system begins to reequilibrate in solution converting dimers to monomers, trimers, tetramers, and so forth. We have observed slow redistribution of oligomers in our previous work, even in the absence of free thiol (see Supporting Information of ref 36). Therefore, a purified oligomer fraction must be stored as a dry solid, or some other approach, such as, encapsulation, must be applied to prevent further ligand exchange.

To conclude, our work on dithiol-induced oligomerization of MPCs provides tools and knowledge for developing controlled synthesis of covalently bound MPCs and nanoparticles for scientific and industrial applications.

\section{ASSOCIATED CONTENT}

\section{S Supporting Information}

The Supporting Information is available free of charge on the ACS Publications website at DOI: 10.1021/acs.jpcc.8b02988.

Calibration curve for converting scanned grayscale value to absorbance; TEM image of $\mathrm{M}$ band of BMM-BPy 1 linked AuMPCs; TEM image of D band of BMM-BPy 1 linked AuMPCs; TEM image of $\mathrm{T}$ band of BMM-BPy 1 linked AuMPCs; TEM image of Q-band of BMM-BPy 1 linked AuMPCs; UV-vis absorption spectrum of each visible band in the PAGE gel; PAGE gel showing bands of $\mathrm{Au}_{\sim 250}(p-\mathrm{MBA})_{n}$ linked with BMM-BPy 1; PAGE gel showing bands of $\mathrm{Au}_{\sim 250}(p-\mathrm{MBA})_{n}$ linked with BMMBPy 1; absorbance of BMM-BPy 1 linked $\mathrm{Au}_{\sim 250}(p$ $\mathrm{MBA})_{n}$ as a function of the PAGE running distance measured from the scanned grayscale image of the PAGE gel; statistics of multimers in the crude product as determined from TEM for two different linker-to-MPC molar ratios; absorbance of BMM-BPy 1 linked $\mathrm{Au}_{\sim 250}(p-\mathrm{MBA})_{n}$ as a function of the PAGE running distance measured from the scanned grayscale image of the PAGE gel (blue), set of Gaussian functions with linear background fitted to data manually (orange), residual of the fit with respect to the experimental data (dark cyan), and molar ratios given in the top-right corner of each panel; schematic presentation of hypotheses and their outcomes; and fraction of MPCs after one-way, random, and independent ligand exchange that have no dithiols (black), one (blue), two (green), three (cyan), four (magenta), and more than four dithiols (dashed red) (PDF)

\section{AUTHOR INFORMATION}

\section{Corresponding Authors}

*E-mail: lauri.lehtovaara@jyu.fi (L.L.).
*E-mail: tanja.m.lahtinen@jyu.fi. Phone: +358 408053697 (T.L.).

ORCID

Eero Hulkko: 0000-0002-7536-5595

Tanja Lahtinen: 0000-0002-1747-6959

Notes

The authors declare no competing financial interest.

\section{ACKNOWLEDGMENTS}

This work was financially supported by the Academy of Finland via projects 269402, 273499, 303753 (L.L.), and 265502, 290677 (E.H.). We acknowledge Prof. Maija Nissinen for the fruitful discussions.

\section{REFERENCES}

(1) Grzelczak, M.; Vermant, J.; Furst, E. M.; Liz-Marzán, L. M. Directed Self-Assembly of Nanoparticles. ACS Nano 2010, 4, 35913605.

(2) Halas, N. J.; Lal, S.; Chang, W.- S.; Link, S.; Nordlander, P. Plasmons in Strongly Coupled Metallic Nanostructures. Chem. Rev. 2011, 111, 3913-3961.

(3) Banik, M.; Rodriguez, K.; Hulkko, E.; Apkarian, V. A. Orientation-Dependent Handedness of Chiral Plasmons on Nanosphere Dimers: How to Turn a Right Hand into a Left Hand. ACS Photonics 2016, 3, 2482-2489.

(4) Homberger, M.; Simon, U. On the Application Potential of Gold Nanoparticles in Nanoelectronics and Biomedicine. Philos. Trans. $R$. Soc., A 2010, 368, 1405-1453.

(5) Nie, S.; Emory, S. R. Probing Single Molecules and Single Nanoparticles by Surface- Enhanced Raman Scattering. Science 1997, $275,1102-1106$

(6) Yampolsky, S.; Fishman, D. A.; Dey, S.; Hulkko, E.; Banik, M.; Potma, E. O.; Apkarian, V. A. Seeing a Single Molecule Vibrate through Time-Resolved Coherent Anti-Stokes Raman Scattering. Nat. Photonics 2014, 8, 650-656.

(7) Watanabe, K.; Menzel, D.; Nilius, N.; Freund, H.-J. Photochemistry on Metal Nanoparticles. Chem. Rev. 2006, 106, 4301-4320.

(8) Brongersma, M. L.; Halas, N. J.; Nordlander, P. Plasmon-Induced Hot Carrier Science and Technology. Nat. Nanotechnol. 2015, 10, 2534

(9) Tapio, K.; Leppiniemi, J.; Shen, B.; Hytönen, V. P.; Fritzsche, W.; Toppari, J. J. Toward Single Electron Nanoelectronics Using SelfAssembled DNA Structure. Nano Lett. 2016, 16, 6780-6786.

(10) Klein, D. L.; McEuen, P. L. An Approach for Electrical Study of Single Nanocrystals. Appl. Phys. Lett. 1996, 68, 2574-2576.

(11) Quinten, M.; Kreibig, U.; Schönauer, D.; Genzel, L. Optical Absorption Spectra of Pairs of Small Metal Particles. Surf. Sci. 1985, $156,741-750$.

(12) Wang, X.; Li, G.; Chen, T.; Yang, M.; Zhang, Z.; Wu, T.; Chen, H. Polymer-Encapsulated Gold-Nanoparticle Dimers: Facile Preparation and Catalytical Application in Guided Growth of Dimeric ZnONanowires. Nano Lett. 2008, 8, 2643-2647.

(13) Kleinman, S. L.; Sharma, B.; Blaber, M. G.; Henry, A.-I.; Valley, N.; Freeman, R. G.; Natan, M. J.; Schatz, G. C.; Van Duyne, R. P. Structure Enhancement Factor Relationships in Single Gold Nanoantennas by Surface-Enhanced Raman Excitation Spectroscopy. J. Am. Chem. Soc. 2013, 135, 301-308.

(14) Cunningham, A.; Mühlig, S.; Rockstuhl, C.; Bürgi, T. Coupling of Plasmon Resonances in Tunable Layered Arrays of Gold Nanoparticles. J. Phys. Chem. C 2011, 115, 8955-8960.

(15) Haynes, C. L.; Van Duyne, R. P. Nanosphere Lithography: A Versatile Nanofabrication Tool for Studies of Size-Dependent Nanoparticle Optics. J. Phys. Chem. B 2001, 105, 5599-5611.

(16) Claridge, S. A.; Liang, H. W.; Basu, S. R.; Fréchet, J. M. J.; Alivisatos, A. P. Isolation of Discrete Nanoparticle - DNA Conjugates for Plasmonic Applications. Nano Lett. 2008, 8, 1202-1206. 
(17) Brousseau, L. C., III; Novak, J. P.; Marinakos, S. M.; Feldheim, D. L. Assembly of Phenylacetylene-Bridged Gold Nanocluster Dimers and Trimers. Adv. Mater. 1999, 11, 447-449.

(18) Dadosh, T.; Gordin, Y.; Krahne, R.; Khivrich, I.; Mahalu, D.; Frydman, V.; Sperling, J.; Yacoby, A.; Bar-Joseph, I. Measurement of the Conductance of Single Conjugated Molecules. Nature 2005, 436, 677-680.

(19) Durocher, S.; Rezaee, A.; Hamm, C.; Rangan, C.; Mittler, S.; Mutus, B. Disulfide-Linked, Gold Nanoparticle Based Reagent for Detecting Small Molecular Weight Thiols. J. Am. Chem. Soc. 2009, 131, 2475-2477.

(20) Tsai, D.-H.; Cho, T. J.; DelRio, F. W.; Gorham, J. M.; Zheng, J.; Tan, J.; Zachariah, M. R.; Hackley, V. A. Controlled Formation and Characterization of Dithiothreitol-Conjugated Gold Nanoparticle Clusters. Langmuir 2014, 30, 3397-3405.

(21) DeVries, G. A.; Brunnbauer, M.; Hu, Y.; Jackson, A. M.; Long, B.; Neltner, B. T.; Uzun, O.; Wunsch, B. H.; Stellacci, F. Divalent Metal Nanoparticles. Science 2007, 315, 358-361.

(22) Gadogbe, M.; Chen, M.; Zhao, X.; Saebo, S.; Beard, D. J.; Zhang, D. Can Para-Aryl-Dithiols Cross-Link Two Plasmonic Noble Nanoparticles as Monolayer Dithiolate Spacers. J. Phys. Chem. C 2015, 119, 6626-6633.

(23) Gang, O. Nanoparticle Assembly: from Fundamentals to Applications: Concluding Remarks. Faraday Discuss. 2016, 186, 529537.

(24) Tsukuda, T.; Häkkinen, H. N. Protected Metal Clusters: From Fundamentals to Applications; Elsevier: Amsterdam, Netherlands, 2015. (25) Jin, R.; Zeng, C.; Zhou, M.; Chen, Y. Atomically Precise Colloidal Metal Nanoclusters and Nanoparticles: Fundamentals and Opportunities. Chem. Rev. 2016, 116, 10346-10413.

(26) Heaven, M. W.; Dass, A.; White, P. S.; Holt, K. M.; Murray, R. W. Crystal Structure of the Gold Nanoparticle $\left[\mathrm{N}\left(\mathrm{C}_{8} \mathrm{H}_{17}\right)_{4}\right]$ $\left[\mathrm{Au}_{25}\left(\mathrm{SCH}_{2} \mathrm{CH}_{2} \mathrm{Ph}\right)_{18}\right]$. J. Am. Chem. Soc. 2008, 130, 3754-3755.

(27) Qian, H.; Eckenhoff, W. T.; Zhu, Y.; Pintauer, T.; Jin, R. Total Structure Determination of Thiolate-Protected $\mathrm{Au}_{38}$ Nanoparticles. J. Am. Chem. Soc. 2010, 132, 8280-8281.

(28) Jadzinsky, P. D.; Calero, G.; Ackerson, C. J.; Bushnell, D. A.; Kornberg, R. D. Structure of a Thiol Monolayer-Protected Gold Nanoparticle at 1.1 A Resolution. Science 2007, 318, 430-433.

(29) Azubel, M.; Koivisto, J.; Malola, S.; Bushnell, D.; Hura, G. L.; Koh, A. L.; Tsunoyama, H.; Tsukuda, T.; Pettersson, M.; Häkkinen, H.; Kornberg, R. D. Electron Microscopy of Gold Nanoparticles at Atomic Resolution. Science 2014, 345, 909-912.

(30) Tvedte, L. M.; Ackerson, C. J. Size-Focusing Synthesis of Gold Nanoclusters with $p$-Mercaptobenzoic Acid. J. Phys. Chem. A 2014, 118, 8124-8128.

(31) Negishi, Y.; Nakazaki, T.; Malola, S.; Takano, S.; Niihori, Y.; Kurashige, W.; Yamazoe, S.; Tsukuda, T.; Häkkinen, H. A Critical Size for Emergence of Nonbulk Electronic and Geometric Structures in Dodecanethiolate-Protected Au Clusters. J. Am. Chem. Soc. 2015, 137, $1206-1212$.

(32) Sakthivel, N. A.; Theivendran, S.; Ganeshraj, V.; Oliver, A. G.; Dass, A. Crystal Structure of Faradaurate-279: $\mathrm{Au}_{279}(\mathrm{SPh}-\mathrm{BBu})_{84}$ Plasmonic Nanocrystal Molecules. J. Am. Chem. Soc. 2017, 139, 15450-15459.

(33) Peterle, T.; Ringler, P.; Mayor, M. Gold Nanoparticles Stabilized by Acetylene-Functionalized Multidentate Thioether Ligands: Building Blocks for Nanoparticle Superstructures. Adv. Funct. Mater. 2009, 19, 3497-3506.

(34) Yao, H.; Yaomura, S. Emergence of Large Chiroptical Responses by Ligand Exchange Cross-Linking of Monolayer-Protected Gold Clusters with Chiral Dithiol. Langmuir 2013, 29, 6444-6451.

(35) Compel, W. S.; Wong, O. A.; Chen, X.; Yi, C.; Geiss, R.; Häkkinen, H.; Knappenberger, K. L., Jr; Ackerson, C. J. Dynamic Diglyme-Mediated Self-Assembly of Gold Nanoclusters. ACS Nano 2015, 9, 11690-11698.

(36) Lahtinen, T.; Hulkko, E.; Sokołowska, K.; Tero, T.-R.; Saarnio, V.; Lindgren, J.; Pettersson, M.; Häkkinen, H.; Lehtovaara, L.
Covalently Linked Multimers of Gold Nanoclusters $\mathrm{Au}_{102}(\mathrm{p}-\mathrm{MBA})_{44}$ and $\mathrm{Au}_{\sim 250}(\mathrm{p}-\mathrm{MBA})_{\mathrm{n}}$. Nanoscale 2016, 8, 18665-18674.

(37) Gürtler, A.; Kunz, N.; Gomolka, M.; Hornhardt, S.; Friedl, A. A.; McDonald, K.; Kohn, J. E.; Posch, A. Stain-Free Technology as a Normalization Tool in Western Blot Analysis. Anal. Biochem. 2013, 433, $105-111$.

(38) Kimura, K.; Sugimoto, N.; Sato, S.; Yao, H.; Negishi, Y.; Tsukuda, T. Size Determination of Gold Clusters by Polyacrylamide Gel Electrophoresis in a Large Cluster Region. J. Phys. Chem. C 2009, 113, 14076-14082.

(39) Ghosh, A.; Hassinen, J.; Pulkkinen, P.; Tenhu, H.; Ras, R. H. A.; Pradeep, T. Simple and Efficient Separation of Atomically Precise Noble Metal Clusters. Anal. Chem. 2014, 86, 12185-12190.

(40) Salassa, G.; Sels, A.; Mancin, F.; Bürgi, T. Dynamic Nature of Thiolate Monolayer in $\mathrm{Au}_{25}(\mathrm{SR})_{18}$ Nanoclusters. ACS Nano 2017, 11, 12609-12614.

(41) Asua, J. M.; McAuley, K. Y. C.; Kim, B. Polymer Reaction Engineering; Blackwell Publishing: Oxford, UK, 2008.

(42) Liu, K.; Nie, Z.; Zhao, N.; Li, W.; Rubinstein, M.; Kumacheva, E. Step-Growth Polymerization of Inorganic Nanoparticles. Science 2010, 329, 197-200.

(43) Carnall, J. M. A.; Waudby, C. A.; Belenguer, A. M.; Stuart, M. C. A.; Peyralans, J. J.-P.; Otto, S. Mechanosensitive Self-Replication Driven by Self-Organization. Science 2010, 327, 1502-1506.

(44) Otto, S.; Furlan, R. L. E.; Sanders, J. K. M. Dynamic Combinatorial Libraries of Macrocyclic Disulfides in Water. J. Am. Chem. Soc. 2000, 122, 12063-12064.

(45) Wang, J.; Xia, H.; Zhang, Y.; Lu, H.; Kamat, R.; Dobrynin, A. V.; Cheng, J.; Lin, Y. Nucleation-Controlled Polymerization of Nanoparticles into Supramolecular Structures. J. Am. Chem. Soc. 2013, 135, 11417-11420.

(46) Sels, A.; Salassa, G.; Pollitt, S.; Guglieri, C.; Rupprechter, G.; Barrabés, N.; Bürgi, T. Structural Investigation of the Ligand Exchange Reaction with Rigid Dithiol on Doped (Pt, Pd) $\mathrm{Au}_{25}$ Clusters. J. Phys. Chem. C 2017, 121, 10919-10926.

(47) Heinecke, C. L.; Ni, T. W.; Malola, S.; Mäkinen, V.; Wong, O. A.; Häkkinen, H.; Ackerson, C. J. Structural and Theoretical Basis for Ligand Exchange on Thiolate Monolayer Protected Gold Nanoclusters. J. Am. Chem. Soc. 2012, 134, 13316-13322. 